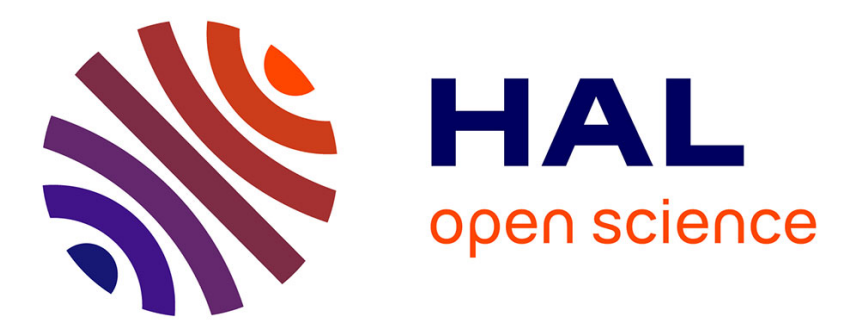

\title{
Structure factor model for understanding the measured backscatter coefficients from concentrated cell pellet biophantoms
}

Emilie Franceschini, Régine Guillermin, Franck Tourniaire, Sandrine Roffino, Edouard Lamy, Jean-François Landrier

\section{To cite this version:}

Emilie Franceschini, Régine Guillermin, Franck Tourniaire, Sandrine Roffino, Edouard Lamy, et al.. Structure factor model for understanding the measured backscatter coefficients from concentrated cell pellet biophantoms. Journal of the Acoustical Society of America, 2014, 135 (6), pp.3620-3631. 10.1121/1.4876375 . hal-01297175

\section{HAL Id: hal-01297175 \\ https://hal.science/hal-01297175}

Submitted on 3 Apr 2016

HAL is a multi-disciplinary open access archive for the deposit and dissemination of scientific research documents, whether they are published or not. The documents may come from teaching and research institutions in France or abroad, or from public or private research centers.
L'archive ouverte pluridisciplinaire HAL, est destinée au dépôt et à la diffusion de documents scientifiques de niveau recherche, publiés ou non, émanant des établissements d'enseignement et de recherche français ou étrangers, des laboratoires publics ou privés. 
Structure Factor Model for understanding the measured backscatter coefficients from concentrated cell pellet biophantoms

Emilie Franceschini, Régine Guillermin, and Franck Tourniaire

Laboratoire de Mécanique et d'Acoustique LMA - CNRS UPR 7051, Aix-Marseille Université, Centrale Marseille, 13402 Marseille Cedex 20, France

Sandrine Roffino

Laboratoire Motricité Humaine Education Sport Santé LAMHESS, Université de Nice Sophia-Antipolis, Nice, France

Edouard Lamy

Laboratoire de Biochimie, Faculté de Pharmacie, Aix-Marseille Université, Marseille, France

Jean-François Landrier

Nutrition, Obésité et Risque Thrombotique, UMR INSERM 1062/INRA 1260, Aix-Marseille Université, Marseille, France

(Dated: September 1, 2014) 


\begin{abstract}
Ultrasonic backscatter coefficient (BSC) measurements were performed on K562 cell pellet biophantoms with cell concentrations ranging from 0.006 to 0.30 in the $10-42 \mathrm{MHz}$ frequency bandwidth. Three scattering models, namely the Fluid-Filled Sphere Model (FFSM), the Particle Model (PM) and the Structure Factor Model (SFM), were compared for modeling the scattering from an ensemble of concentrated cells. A parameter estimation procedure was developed in order to estimate the scatterer size and relative impedance contrast that could explain the measured BSCs from all the studied cell concentrations. This procedure was applied to the BSC data from K562 cell pellet biophantoms in the 10-42 MHz frequency bandwidth and to the BSC data from Chinese Hamster Ovary cell pellet biophantoms in the 26-105 MHz frequency bandwidth given in [Han et al, "Ultrasonic backscatter coefficient quantitative estimates from high-concentration Chinese hamster ovary cell pellet biophantoms", J. Acoust. Soc. Am. 130, 4139-4147 (2011)]. The data fitting quality and the scatterer size estimates show that the SFM was more suitable than the PM and the FFSM for modeling the responses from concentrated cell pellet biophantoms.
\end{abstract}

PACS numbers: 43.80.Cs, 43.80.Qf, 43.35.Bf

Keywords: quantitative ultrasound, structure factor, cell pellet, ultrasound backscatter, ultrasound tissue characterization 


\section{INTRODUCTION}

Quantitative ultrasound (QUS) techniques for determining the tissue microstructure are based on the frequency-based analysis of the signals backscattered from biological tissues. One approach consists in fitting the measured backscatter coefficient (BSC) from biological tissues to an estimated BSC using an appropriate theoretical scattering model. The most frequently used theoretical scattering model is the spherical Gaussian model developed by Lizzi $^{1,2}$ that describes the tissue as a random inhomogeneous continuum with impedance fluctuations. The spherical Gaussian model yields two tissue properties: the average scatterer size and the acoustic concentration (i.e., the product of the scatterer number density by the square of the relative impedance difference between the scatterers and the surrounding medium). This approach has been used to assess the response to therapy ${ }^{3}$ and to differentiate between diseased and healthy tissue or to detect cancer tumors, for the eye ${ }^{4}$, the prostate $^{5}$, the breast ${ }^{6,7}$, and cancerous lymph nodes. ${ }^{8}$ Another class of theoretical scattering model describes the tissue as randomly distributed discrete scatterers using the Fluid-Filled Sphere Model ${ }^{9,10,36}$ (FFSM) or the concentric sphere model. ${ }^{10,12-14,36}$ In the aforementioned models (spherical gaussian model, FFSM and concentric sphere model), the scatterers are assumed to be independently and randomly distributed (i.e., to have a low scatterer concentration) and multiple scattering is neglected (in line with the Born approximation). Under these hypotheses, the power of the backscattered signals increases linearly with the scatterer concentration and depends on the size and acoustic properties of the tissue scattering structures. This linear relationship has been used to monitor the scatterer size and concentration. However, the assumption of randomly distributed scatterers may not hold in tumors with densely packed cells. ${ }^{17}$ A model adapted to dense medium is the Structure Factor Model (SFM) used in blood characterization. ${ }^{18,19}$ The SFM is based on the assumption that at high scatterer concentrations, interference effects are mainly caused by correlations between the spatial positions of individual scatterers, i.e., caused by coherent scattering. The SFM sums the contributions from individual cells and models the cellular interaction by a statistical 
mechanics structure factor, which is defined as the Fourier transform of the spatial distribution of the cells. ${ }^{18,19}$ The low frequency limit of the SFM is the Particle Model (PM) using the low frequency limit of the structure factor. The low frequency limit of the structure factor is by definition the packing factor. ${ }^{20}$ The packing factor is a measure of orderliness in the spatial arrangement of cells. It depends only on the cell concentration (but not on the frequency). Experiments on tissue-mimicking phantoms were recently performed by our group ${ }^{21}$ to compare the SFM with other classical scattering models (spherical gaussian model, elastic sphere ${ }^{25}$ and PM). This study showed that the SFM was the most suitable theoretical scattering model for dealing with concentrated media such as densely packed cells in tumors.

In parallel with this work on QUS techniques, experimental studies were also conducted to understand and identify the cellular scattering sources. Identifying the scattering sites will lead to the improvement of the theoretical scattering models. Baddour et $a l^{22,23}$ and Falou et $a l^{24}$ performed measurements of high frequency (12-57 MHz) ultrasound BSC responses from individual cells and modeled a cell as a single spherical scatterer with uniform acoustic properties. The BSC measurements were compared to theoretical BSC predictions from a fluid sphere model $^{9}$ or from an elastic sphere model. ${ }^{25}$ It was found that the BSC from an isolated cell was best modeled as a fluid sphere having the whole cell size. ${ }^{24}$ Taggart et $a l^{26}$ conducted high frequency ultrasound measurements on cell pellets (i.e. highly packed cells) made up of mono- and multi-nucleated cells or isolated nuclei. This study suggests that the integrated BSCs were correlated with the size of the nuclei. Teisseire et $a l^{13}$ and Han et $a l^{14}$ developed cell pellet biophantoms that consist of identical cells embedded in a plasmathrombin supportive background with various cell concentrations ranging from 0.0017 to 0.63. The concentrated biophantoms mimic densely packed cells with controlled cell volume fractions and are simplified versions of real tissue since only a single cell line is considered. The BSC estimates from the biophantoms were fitted with the concentric sphere model ${ }^{12}$ in the 26-105 MHz frequency bandwidth. At low cell concentrations $(\leq 0.026)$, the estimated whole cell radii agree well with the true whole cell radii, but not at high cell concentrations 
(>0.096). ${ }^{14}$ A nonlinear relationship between the BSC amplitude and the cell concentration was also observed below $50 \mathrm{MHz}$, which might be attributed to the coherent scattering. ${ }^{14}$ This work on concentrated cell pellet biophantoms suggests that the concentric sphere model becomes less reliable as the cell concentration increases. ${ }^{14}$

The aim of this study was to use the SFM to go further in the understanding of the measured BSCs from cell pellet biophantoms. The SFM was modified to introduce the polydispersity by using a local monodisperse approximation. ${ }^{15,16}$ The FFSM and the PM were also examined in both monodisperse and polydisperse modeling cases for the comparison with the SFM. Ultrasonic backscatter measurements were performed at frequencies ranging from 10 to $42 \mathrm{MHz}$ on biophantoms. These biophantoms consisted of human leukemia K562 cells trapped in a mixture of plasma and thrombin with different cell concentrations ranging from 0.006 to 0.30 . A parameter estimation procedure was developed in order to estimate the scatterer size and relative impedance contrast that could explain the measured $B S C_{m e a s}$ from all the studied cell concentrations using the FFSM, PM and SFM. This procedure was applied to our BSC data from K562 cell pellet biophantoms in the 10-42 MHz frequency bandwidth and to the BSC data from Chinese Hamster Ovary (CHO) cell pellet biophantoms in the 26-105 MHz frequency bandwidth given in Ref. 14. The scatterer sizes estimated using our parameter estimation procedure were compared to the true cellular features to identify the scattering sites. The ability of the FFSM, PM and SFM to model the measured BSCs from concentrated cell pellet biophantoms with a single set of structural and acoustic parameters was discussed.

\section{ULTRASOUND SCATTERING THEORY}

In the following, it is assumed that shear wave propagation and wave mode conversion are neglected so that only compressional waves are taken into account. The surrounding medium is acoustically described as a homogeneous fluid medium, characterized by a sound speed $c_{0}$ and a density $\rho_{0}$. This section presents the BSC modeling for an ensemble of cells 
using the FFSM, SFM and PM. For all three models, the formulations were written for monodisperse and polydisperse spheres.

\section{A. The Fluid-Filled Sphere Model (FFSM)}

An isolated cell was modeled as a fluid-filled sphere representing the whole cell or the nucleus. The exact solution for the scattering of a plane wave of wave number $k$ by a fluidfilled sphere of radius $r$, sound speed $c$ and density $\rho$ embedded in a fluid medium can be calculated using the Anderson model. ${ }^{9}$ In this study, we assumed weak scattering contrast such that the differential backscattering cross section from a single fluid-filled sphere $\sigma_{b}$ was calculated using the following expression:

$$
\sigma_{b}(k, r)=\frac{k^{4} V_{s}^{2} \gamma_{z}^{2}}{4 \pi^{2}}\left(3 \frac{\sin (2 k r)-2 k r \cos (2 k r)}{(2 k r)^{3}}\right)^{2},
$$

where $V_{s}$ is the sphere volume and $\gamma_{z}$ is the relative impedance difference between the cells

and the surrounding medium $\gamma_{z}=\frac{c \rho-c_{0} \rho_{0}}{c_{0} \rho_{0}}$. By considering an ensemble of identical fluid spheres independently and randomly distributed, the theoretical BSC using the FFSM can be written as:

$$
\operatorname{BSC}_{F F S M}^{\text {monod }}(k)=m \sigma_{b}(k, r)
$$

where $m$ is the number density related to the sphere concentration $\phi$ as $m=\phi / V_{s}$.

However, even if the same cell line is used in the biophantom, the cells are not identical in size and the BSC is affected by the scatterer size distribution. By considering a mixture of spheres differing only in size, the theoretical BSC using the FFSM can be expressed as:

$$
B S C_{F F S M}^{\text {polyd }}(k)=m \int_{0}^{\infty} p(r) \sigma_{b}(k, r) d r
$$

where $p(r)$ is the sphere radius probability distribution function (i.e., the probability that the sphere radius takes the value $r$ ). The number density $m$ is related to the total sphere concentration $\phi$ as

$$
m=\frac{\phi}{(4 / 3) \pi \int_{0}^{\infty} p(r) r^{3} d r}
$$


Note that the dependence of $\sigma_{b}$ on $r$ in Eq. (2) is usually not written; this dependence is necessary here for understanding the mathematical integration operation in Eq. (3). Figure ??(a) shows examples of theoretical BSCs computed with the FFSM in both monodiperse and polydiperse cases. We considered a monodisperse distribution of sphere radius $6.5 \mu \mathrm{m}$ and a polydisperse distribution with a Gaussian size distribution of $6.5 \pm 1.5 \mu \mathrm{m}$. In both monodisperse and polydisperse cases, the relative impedance contrast of the spheres was equal to $\gamma_{z}=0.05$ and three sphere concentrations of $0.006,0.06$ and 0.3 were studied. One can notice from Fig. ??(a) that the size variance influences mainly the amplitude of the first BSC dip (in the example given at the frequency around $87 \mathrm{MHz}$ ). Whatever the sphere concentrations of $0.006,0.06$ and 0.3 in the monodisperse case (or in the polydisperse case), the frequency dependence of the BSC curves is identical for a given scatterer size $r$. It is due to the fact that the BSC frequency dependence for the FFSM depends only on $\sigma_{b}(k, r)$.

\section{B. The Structure Factor Model (SFM) and the Particle Model (PM)}

\section{Monodisperse case}

The $\mathrm{SFM}^{18}$ is based on the assumption that at high scatterer concentrations, interference effects are mainly caused by correlations between the spatial positions of individual scatterers. In comparison with the FFSM described in Eq. (2), the SFM considers the interference effects relatively easily by replacing the single-particle backscattering contribution $\sigma_{b}(k, r)$ by the product $\sigma_{b}(k, r) S(k, r)$, where $S(k, r)$ is the structure factor depending on the scatterer concentration $\phi$ and the pattern of the spatial arrangement of the scatterers. By considering an ensemble of identical fluid spheres of radius $r$, the theoretical BSC for the SFM formulation is given by:

$$
\operatorname{BSC}_{S F M}^{\text {monod }}(k)=m \sigma_{b}(k, r) S(k, r, \phi),
$$

where the differential backscattering cross section $\sigma_{b}$ is calculated using Eq. (1). Note that there is no simple analytical expression of the structure factor for a complex spatial 
positioning of particles as occurs in the case of aggregates of particles. However, for an ensemble of identical hard (i.e. non deformable) spheres that are homogeneously distributed, the structure factor depends on the sphere radius and the sphere concentration, and its analytical expression can be obtained as established by Wertheim. ${ }^{27}$ The analytical expression of the structure factor used here was computed from equations (A1)-(A4) in Ref. 21. The structure factor has an influence on the BSC frequency dependency and amplitude, as observed by plotting the structure factor versus the product $k r$, where $r$ is the sphere radius (see Fig. 14(a) of Ref. 21). For example, in the case of a sphere concentration of 0.15, the structure factor ranges between 0.30 and 0.77 for $k r$ ranging between 0 and 1 (see the green solid line in Fig. 14(a) in Ref. 21).

In the low frequency limit, the structure factor tends towards a constant value $S(k) \rightarrow$ $S(0)=W$ called the packing factor. ${ }^{20}$ The most commonly used expression for the packing factor is based on the Percus-Yevick pair-correlation function for identical, hard (i.e. nondeformable) and radially symmetric particles. The Perkus-Yevick packing factor $W_{P Y}$ is linked to the scatterer concentration $\phi$ as: ${ }^{20}$

$$
W_{P Y}=\frac{(1-\phi)^{4}}{(1+2 \phi)^{2}}
$$

Thus, in the low frequency limit, by considering an ensemble of identical fluid spheres of radius $r$, the theoretical $\mathrm{BSC}$ for the $\mathrm{PM}$ formulation is given by:

$$
B S C_{P M}^{\text {monod }}(k)=m \frac{(1-\phi)^{4}}{(1+2 \phi)^{2}} \sigma_{b}(k, r)
$$

\section{Polydisperse case}

By considering a mixture of hard spheres differing only in size, the SFM expression involves the partial structure factor, as in Pedersen ${ }^{15,16}$ in the field of small-angle scattering or in Berger $e t a l^{28}$ in the field of ultrasound. Since the computation of the partial structure factor is not straightforward, ${ }^{15,16,28}$ approximations for polydisperse systems are often used 
to fit the scattering data. In this study, we used a local monodisperse approximation. ${ }^{15,16}$ It is assumed that a scatterer of a certain size is always surrounded by scatterers of the same size. Under this hypothesis, the polydisperse system is approximated by many subsystems in which the particles are monodisperse. The scattering of the polydisperse medium is calculated as the sum of the scattering from the monodisperse subsystems weighted by the sphere radius probability distribution function $p(r)$. The theoretical BSC for a mixture of spheres differing only in size is thus given by:

$$
B S C_{S F M}^{p o l y d}(k)=m \int_{0}^{\infty} p(r) \sigma_{b}(k, r) S(k, r, \phi) d r
$$

where $S(k, r, \phi)$ is the monodisperse structure factor for an equivalent system, consisting only of particles of radius $r$ with a fixed total concentration $\phi$.

In the low frequency limit, the theoretical BSC for the PM formulation in the polydisperse case is given by:

$$
B S C_{P M}^{p o l y d}(k)=m \frac{(1-\phi)^{4}}{(1+2 \phi)^{2}} \int_{0}^{\infty} p(r) \sigma_{b}(k, r) d r
$$

Figure ??(b) and (c) shows examples of theoretical BSCs computed with the SFM and the PM in both monodisperse and polydisperse cases with the same sphere configuration presented as the one presented in section II.A. One can notice from Fig. ??(b) and (c) that the BSC amplitude and frequency dependence computed with the PM and the SFM differ for the higher concentrations of 0.06 and 0.3. Indeed, the BSC frequency dependence computed with the PM (or with the SFM, respectively) depends on $\sigma_{b}(k, r)$ (on $\sigma_{b}(k, r)$ and $S(k, r, \phi)$, respectively). That is why the frequency dependence of the BSC curves is identical with the PM (and is different with the SFM) for the sphere concentrations of 0.006 , 0.06 and 0.3 . 


\section{METHODS}

\section{A. Preparation of the K562 cell pellet biophantoms}

The preparation of the cell pellet biophantoms was adapted from Teisseire et $a l^{13}$ and is presented here.

Human leukemia K562 cells were obtained from the European Collection of Cell Cultures (Salisbury, UK). K562 cells were grown in Roswell Park Memorial Institute 1640 medium (Life Technologies, Saint Aubin, France) supplemented with 10\% fetal calf serum (PAA, Velizy-Villacoublay, France), $25 \mathrm{mM}$ HEPES and 100 units of penicillin and $100 \mu \mathrm{g}$ of streptomycin (Life Technologies). Cells were maintained at $37^{\circ} \mathrm{C}$ in a humidified atmosphere of $5 \% \mathrm{CO} 2$ and $95 \%$ air.

The size distributions of K-562 nuclei were estimated from optical microscopy images of extracted nuclei. Nuclei were extracted using the protocol of Greenberg and Bender, ${ }^{29}$ which is briefly recalled in the following. $10^{7}$ cells were washed twice with $50 \mathrm{~mL}$ of ice-cold PBS and pelleted by centrifugation for $5 \mathrm{~min}$ at $4^{\circ} \mathrm{C}$ and $500 \mathrm{~g}$. The cell pellet was then gently vortexed and $4 \mathrm{~mL}$ of lysis buffer $(10 \mathrm{mM}$ Tris-HCl pH 7.4, $10 \mathrm{mM} \mathrm{NaCl}, 3 \mathrm{mM}$ $\mathrm{MgCl} 2,0.5 \% \mathrm{NP}-40(\mathrm{v} / \mathrm{v}))$ were added under constant vortexing. After the whole volume of lysis had been dispensed, the cells were vortexed at maximum speed for $10 \mathrm{sec}$ and then incubated on ice for $5 \mathrm{~min}$. At this stage, a few microliters of the lysate were placed on a hemocytometer and observed under microscope to check whether nuclei have been released and are free of cytoplasmic material. The cells were then centrifuged again as previously and the supernatant was discarded. $4 \mathrm{~mL}$ of ice-cold lysis buffer were added under gentle vortexing as described before, and the nuclei were spun for $5 \mathrm{~min}$ at $4^{\circ} \mathrm{C}$ and $500 \mathrm{~g}$. The pellet was then resuspended in 100-300 $\mu \mathrm{L}$ of ice-cold glycerol buffer (50 mM Tris- $\mathrm{HCl} \mathrm{pH}$ 8.3, $5 \mathrm{mM} \mathrm{MgCl}$, $0.1 \mathrm{mM} \mathrm{EDTA,} \mathrm{40 \%} \mathrm{(v/v)} \mathrm{glycerol),} \mathrm{and} \mathrm{diluted} 20$ times with ice-cold PBS.

The radius probability distribution functions estimated for extracted nuclei and whole cells are given in Fig. ??. Measurements were made using a calibrated optical microscope 
on 200 extracted nuclei and 120 whole cells randomly selected. The radius probability distribution functions for nuclei and whole cells were well approximated by Gaussian distributions. The mean nuclear and whole cell radii were found to be equal to $4.18 \pm 0.43 \mu \mathrm{m}$ and $6.34 \pm 0.94 \mu \mathrm{m}$, respectively. Because of the polydispersity in the cell radius, the whole cell volume was calculated using a corrected sphere radius $r_{c}^{c o r}$ of $6.48 \mu \mathrm{m}$, which is determined by the expression $\left(\operatorname{mean}\left(r_{c}^{3}\right)\right)^{1 / 3}$, where $r_{c}$ is the measured cell radius by optical microscopy. ${ }^{14}$

Biophantoms with different cell concentrations of $0.006,0.03,0.06,0.12,0.18,0.24$ and 0.30 were studied. For each biophantom concentration studied, three samples of $100 \mu \mathrm{L}$ of the cell culture were withdrawn from the cell medium in order to estimate the mean cell concentration using the Scepter ${ }^{\mathrm{TM}} 2.0$ cell counter (Millipore, Molsheim, France). A known number of cells were then suspended in a $1.5 \mathrm{~mL}$ plastic tube in a mixture consisting of $272 \mu \mathrm{L}$ of human plasma (obtained after collection of blood from volunteers in citrated tubes), $3 \mu \mathrm{L}$ of $1 \mathrm{M} \mathrm{CaCl} 2$ and $25 \mu \mathrm{L}$ of thrombin $(6 \mathrm{U} / \mathrm{mL}$ in bi-distilled water, Sigma-Aldrich, Saint Quentin Fallavier, France) to reach the desired concentration of the cell pellet biophantoms. After homogeneization with a pipette tip, the suspension was transferred into a well of a 8-well Nunc Lab-Tek II Chamber Slide System (Dominique Dutscher, Brumath, France) and plasma was allowed to coagulate for $1 \mathrm{~h}$ at room temperature under agitation $(70 \mathrm{rpm})$ on a Stuart ${ }^{\mathrm{TM}}$ Scientific Gyro rocker. The chamber slide was then placed in a plastic dish and immerged in PBS to allow ultrasound measurement. All the cell pellet preparation procedure was repeated twice.

After the ultrasound measurement, cell pellet biophantoms were fixed in a $10 \%(\mathrm{w} / \mathrm{v})$ formalin solution for $48 \mathrm{~h}$. The cell pellets were then removed from the chamber slide and embedded in paraffin to be sectioned. The sections were then stained with hematoxylin and eosin (HE) to verify that the cell spatial distribution was homogeneous (see Fig. ??). 


\section{B. Ultrasound data acquisition}

US measurements were acquired using a Vevo 770 high frequency ultrasound system (Visualsonics Inc, Toronto, Canada). Two RMV 710 and RMV 703 probes were used in B-mode. For the RMV 710 and the RMV 703 probes, the oscillating single-element focused circular transducers had center frequencies of 20 and $30 \mathrm{MHz}$ with $-6 \mathrm{~dB}$ bandwidths of 10-32 and 18-42 MHz, focuses of 15 and $10 \mathrm{~mm}$ and f-numbers of 2.1 and 2.5, respectively. We acquired RF data from this scanner at a sampling frequency of $500 \mathrm{MHz}$ with 8 bit resolution with a Gagescope model CS11G8 acquisition board.

The probe focus was positioned at a distance of $1 \mathrm{~mm}$ below the PBS/cell pellet biophantom interface. A translation stage (Physik Instrumente, model M-403.4PD, Karlsruhe, Germany) controlled the probe motion. Six B-mode images were constructed from acquired RF echoes by translating the probe every $600 \mu \mathrm{m}$. Examples of US B-mode scans obtained with the $20-\mathrm{MHz}$ center frequency probe are provided in Fig. ??. For around 140 vertical lines at the center of each B-mode image, echoes were selected in the focal zone with a rectangular window of $d=0.75 \mathrm{~mm}$, and the power spectra of the backscattered RF echoes were then averaged to provide $P_{\text {meas }}$. This procedure was repeated twice with the two probes at each biophantom concentration studied.

\section{Attenuation and BSC measurements}

The attenuation coefficients of the cell pellet biophantoms were determined by using a standard substitution method. The Vevo 770 US scanner equipped with the $20-\mathrm{MHz}$ center frequency probe was used in M-mode for reflection measurements. The measured cell pellet attenuation $\alpha_{p h}$ (in $\left.\mathrm{dB} / \mathrm{mm}\right)$ was computed as:

$$
\alpha_{p h}(f)=\alpha_{P B S}(f)+\frac{20}{2 D} \log _{10}\left(\frac{S_{r e f}(f)}{S_{p h}(f)}\right)
$$

where $\alpha_{P B S}$ is the frequency-dependent attenuation of the PBS, which was taken to be similar to water, $2.1715 \times 10^{-4} \mathrm{~dB} \mathrm{MHz}-2 \mathrm{~mm}^{-1}$ at $20{ }^{\circ} \mathrm{C} .{ }^{30} f$ is the frequency and $D$ is the 
thickness of the cell pellet. $S_{p h}$ and $S_{r e f}$ are the amplitude spectra of the reflected signals from the well base surface with and without the presence of the cell pellet. Assuming that the cell pellet attenuation increases linearly with the frequency $\left(\alpha_{p h}(f)=\alpha_{p h_{0}} f\right)$, a linear regression analysis provided the attenuation coefficient $\alpha_{p h_{0}}$ in $\mathrm{dB} \mathrm{MHz}^{-1} \mathrm{~mm}^{-1}$. Averaged values obtained for 10 locations in the pellet well were 0.0098, 0.0184, 0.0215, 0.0202 and $0.0280 \mathrm{~dB} \mathrm{MHz}^{-1} \mathrm{~mm}^{-1}$ for cell pellet concentrations of $0.06,0.12,0.18,0.24$ and 0.30 , respectively.

The measured BSC values reported in this study were computed using the reference phantom technique. ${ }^{31,32}$ This technique consists in using a reference scattering medium instead of a perfect flat reflector, on condition that the BSC of the reference scattering medium is known or can be determined. The reference scattering medium makes it possible to compensate the measured backscattered power spectrum $\overline{P_{\text {meas }}}$ for the electromechanical system response and the depth-dependent diffraction and focusing effects caused by the ultrasound beam. The reference scattering medium used was a mixture of distilled water, $2 \%(\mathrm{w} / \mathrm{w})$ agar powder (A9799, Sigma Aldrich, France), and 1\% (w/w) of polyamide microspheres with a radius of $2.5 \mu \mathrm{m}$ (orgasol 2001 UD NAT1, Arkema, France). The sample is easy to prepare and to handle, and the scattering process occurring in an ensemble of identical solid microspheres at a very low concentration of $1 \%$ (dilute medium) has been well documented using the Faran model. ${ }^{25}$ Echoes from the reference scattering medium were acquired and windowed as with the cell pellet biophantoms, and their power spectra were averaged to obtain $\overline{P_{\text {ref }}}$. The measured BSC was thus computed as follows ${ }^{31,32}$

$$
B S C_{\text {meas }}(k)=B S C_{\text {ref }}(k) \frac{\overline{P_{\text {meas }}(k)}}{\overline{P_{\text {ref }}(k)}} e^{4 d\left(\alpha_{p h_{0}}-\alpha_{p h_{r e f}}\right) k \frac{c}{2 \pi}}
$$

where $B S C_{r e f}$ is the theoretical BSC of the reference sample given by Eq. (2) in Ref. 21 using the Faran model, ${ }^{25}$ and $\alpha_{p h_{0}}$ and $\alpha_{p h_{r e f}}$ are the predetermined attenuation coefficients of the cell pellet biophantoms and of the reference phantoms. The value of $\alpha_{p h_{r e f}}$ was equal to $0.004 \mathrm{~dB} \mathrm{MHz}^{-1} \mathrm{~mm}^{-1}$. 


\section{Parameter estimation}

The objective of this study was to identify the cell structures responsible for scattering. In that aim, we estimated the scatterer radius $r$ and its relative impedance contrast $\gamma_{z}$ that could explain the measured $B S C_{\text {meas }}$ from all the studied cell concentrations. Indeed, if a unique scattering model can explain the frequency dependence and magnitude of the $B S C_{\text {meas }}$ data for all the cell concentrations, one could expect to identify the cell structures responsible for scattering. In the following, we will assume that the true cell radius mean (measured by optical microscopy) $r_{c}^{c o r}=6.48 \mu \mathrm{m}$ and the cell concentrations $\phi_{c}=(0.006,0.06$, $0.12,0.18,0.24,0.3)$ are known a priori. By considering that the cell number density is equal to the sphere number density, the fluid sphere concentration $\phi$ was calculated in the monodisperse case as $\phi=\left(r / r_{c}^{c o r}\right)^{3} \phi_{c}$ and in the polydisperse case as:

$$
\phi=\phi_{c} \frac{\int p(r) r^{3} d r}{r_{c}^{c o r} 3}
$$

In the monodisperse case, the unknown parameters were $r$ and $\gamma_{z}$. In the polydisperse case, the sphere radii were assumed to have a Gaussian distribution $p(r)$ with a mean radius $\bar{r}$ and a standard deviation $\mu$. The unknown parameters were thus $\bar{r}, \mu$ and $\gamma_{z}$. For the FFSM, PM and SFM in both monodisperse and polydisperse cases, the unknown parameters were determined by minimizing the cost function $F$, which synthesizes the seven measurements with the seven biophantom concentrations $\left(\phi_{c_{i=1 \ldots 7}}=0.006,0.03,0.06,0.12,0.18,0.24\right.$ and $0.3)$ over the wavenumbers $k_{j}(j=1 \cdots M)$ in the frequency bandwidth studied:

$$
F=\frac{1}{N} \sum_{i=1}^{N} \frac{\sum_{j}\left\|B S C_{\text {meas }}\left(k_{j}, \phi_{c_{i}}\right)-B S C_{\text {theo }}\left(k_{j}, \phi_{c_{i}}\right)\right\|^{2}}{\sum_{j}\left\|B S C_{\text {meas }}\left(k_{j}, \phi_{c_{i}}\right)\right\|^{2}},
$$

where $N$ is the number of studied cell concentrations (here $N=7$ ). Note that the dependence of BSC on $\phi_{c_{i}}$ is usually not written; this dependence is necessary here for understanding the summation over $i$. In the monodisperse case, we employed a routine fminsearch in MATLAB (The Mathworks Inc., Natick, MA), i.e., a Nelder-Mead simplex method, to minimize the cost function $F$, whereas in the polydisperse case, we used a routine fmincon in MATLAB, with the constraint conditions that $0 \leq \bar{r} \leq 60 \mu \mathrm{m}, 0 \leq \mu \leq 1,0 \leq \gamma_{z} \leq 1$ and that the minimum 
value of $r$ was positive.

\section{SCATTERING FROM THE K562 CELL PELLET BIOPHANTOMS}

\section{A. Results}

Figure ??(a) gives examples of the $B S C_{\text {meas }}$ versus frequency curves measured with the $20-\mathrm{MHz}$ and $30-\mathrm{MHz}$ center frequency probes and averaged over the six measurements (corresponding to the six acquired B-mode images as described in section III.B). The experimental study (i.e. the preparation of the cell pellet biophantoms as well as the corresponding US acquisition) was repeated twice at each biophantom concentration to verify the measurement repeatability. The $B S C_{\text {meas }}$ averaged over the six measurements for the two studies are shown in Fig. ??(b) and (c). Standard deviations are not shown to enhance reading. The averaged $B S C_{\text {meas }}$ measured in the two studies have similar frequency dependence and magnitude, showing good repeatability. At all the studied concentrations, the $B S C_{\text {meas }}$ measured with the two probes in the 18-32 MHz frequency bandwidth were similar. This means that the results were not influenced by the system transfer function.

Tables I and II (see lines 1-3) report the scatterer radius $r^{*}$ and the relative impedance contrast $\gamma_{z}^{*}$ estimated by the three models FFSM, PM and SFM in the monodisperse and the polydisperse cases, respectively. Also given in Tables I and II are the corresponding normalized errors to quantify the goodness of fit. The normalized errors were calculated by evaluating the cost function (given by Eq. 13) at the values $r^{*}\left(\right.$ or $\bar{r}^{*} \pm \mu^{*}$ ) and $\gamma_{z}^{*}$. The standard deviation $\mu^{*}$ estimated with the polydisperse FFSM was equal to 0 so that the estimated sphere radius and impedance contrast were identical in the monodisperse and polydisperse cases. Normalized errors were smaller with the polydisperse SFM and larger with the FFSM. Figure ?? displays $B S C_{\text {meas }}$ measured for different cell concentrations, as well as the BSCs results with the polydisperse FFSM and the polydisperse PM calculated by assuming the estimated scatterer size $\bar{r}^{*} \pm \mu^{*}$ and impedance contrast $\gamma_{z}^{*}$ (see values in Table II lines 1-2). One can notice large differences between measured $B S C_{\text {meas }}$ and estimated 
FFSM curves, even at low concentrations $\phi_{c} \leq 0.12$. So the FFSM cannot model the $B S C_{\text {meas }}$ amplitude behavior for all concentrations. The PM provides satisfactory fittings of the $B S C_{\text {meas }}$ for cell concentrations $\phi_{c}<0.18$. As the cell concentration increases, the PM is not able to model the $B S C_{\text {meas }}$ amplitude and frequency dependency. Figure ?? shows the BSCs curves with the SFM in both monodisperse and polydisperse cases calculated by assuming the estimated scatterer size $\bar{r}^{*} \pm \mu^{*}$ and impedance contrast $\gamma_{z}^{*}$ (see values in Tables I and II lines 3). It is clear from these figures that the polydisperse SFM provides the best fits of the $B S C_{\text {meas }}$ for all cell concentrations.

To make the reading of these results easier, Fig. ?? shows the measured BSCs magnitude averaged in the frequency bandwidth (from 10 to $28 \mathrm{MHz}$ and from 10 to $42 \mathrm{MHz}$ ) as a function of the cell concentration. The standard deviations are calculated based on the six measurements (performed every $600 \mu \mathrm{m}$ ) on the same cell pellet biophantoms. Note that the $B S C_{\text {meas }}$ is not linearly proportional to concentration. For the averaged $B S C_{\text {meas }}$ in the 10-28 MHz frequency bandwidth shown in Fig. ??(a), the $B S C_{\text {meas }}$ magnitude increases with increasing concentration between 0.006 and 0.12 , then decreases with increasing concentration between 0.12 and 0.30 . The averaged $B S C_{\text {meas }}$ in the $10-42 \mathrm{MHz}$ frequency bandwidth are in the same range of values for concentrations between 0.18 and 0.30 (see Fig. ??(b)). Also plotted in Fig. ?? are the averaged BSCs computed with the FFSM, PM and SFM using the estimated parameters given in Tables I and II (lines 1-3). The FFSM showed no agreement with the experimental data. Good agreement was obtained at low cell concentrations $\phi_{c} \leq 0.12$ but not for the higher concentrations of 0.24 and 0.30 , for the PM in both monodisperse and polydisperse cases and for the monodisperse SFM, in both 10-28 $\mathrm{MHz}$ and 10-42 MHz frequency ranges. The polydisperse SFM was the model showing the best agreement with the experimental data. 


\section{B. Discussion}

For the polydisperse SFM giving the smallest normalized errors, the estimated radius of the fluid sphere was found equal to $6.40 \mu \mathrm{m}$ and agrees well with the true measured radius of the K562 whole cells, with a relative error around 1.2\%. Concerning the acoustic parameters, the surrounding medium was assumed to be plasma-like, with $c_{0}=1547 \mathrm{~m} / \mathrm{s}$ and $\rho_{0}=1.021$ $\mathrm{g} / \mathrm{mL} .^{33}$ By assuming that the cytoplasm occupies $71 \%$ of the K562 cell volume and that the cytoplasm contains $80-90 \%$ of water, ${ }^{34}$ the cell was thus expected to have water-like acoustic parameters, i.e. $c \approx 1500 \mathrm{~m} / \mathrm{s}, \rho \approx 1$, and $\gamma_{z} \approx 0.05$. A good match was thus obtained between the estimated and expected relative impedances using the polydisperse SFM (see Table II line 3).

The nonlinear relationship between the BSC amplitude and the cell concentration observed in Fig. ?? was correctly modeled with the SFM but not with the FFSM and PM. Since the FFSM assumes that the BSC is linearly proportional to scatterer concentration, it was expected that the FFSM would give the largest errors. Even if the PM can model a nonlinear relationship between the BSC magnitude and the concentration, it was insufficient to explain the $B S C_{\text {meas }}$ behaviors, as shown in Fig. ??. The main explanation for this lack of consistency is that the PM is only effective at low frequencies and loses its applicability in the large frequency range of $10-42 \mathrm{MHz} .^{21}$ To confirm this hypothesis, the estimation procedure was performed with the PM in a low and narrow frequency bandwidth of 10-15 MHz. The estimated parameters were equal to $r^{*}=6.67 \mu \mathrm{m}$ and $\gamma_{z}^{*}=0.060$ in the monodisperse case, and $\bar{r}^{*}=6.66 \mu \mathrm{m}, \mu^{*}=0.059 \mu \mathrm{m}$ and $\gamma_{z}^{*}=0.059$ in the polydisperse case. Both radius and relative impedance contrast estimated in the low 10-15 MHz frequency bandwidth gave better results than those estimated in the 10-42 MHz frequency bandwidth (see Tables I and II lines 2). In the low 10-15 MHz frequency range, the relative error for the radius estimated with the PM was around $2.7 \%$ (against a relative error around $37.8 \%$ in the $10-42$ $\mathrm{MHz}$ frequency bandwidth). Figure ?? represents the averaged $B S C_{\text {meas }}$ in the $10-15 \mathrm{MHz}$ frequency bandwidth versus cell concentrations. The PM provided good agreement with the 
experimental data in this case.

The good quality of the fitted BSC curves (see Fig. ??) as well as the good agreement between the estimated scatterer radius and the true whole cell radius reveals that the polydisperse SFM was more relevant that the FFSM and PM for modeling the responses from concentrated biophantoms in the studied 10-42 MHz frequency bandwidth. This finding also implies that a fluid sphere with the size of the whole cell and uniform acoustic properties is sufficient to model an isolated cell in this frequency range.

\section{SCATTERING FROM CHINESE HAMSTER OVARY (CHO) CELL PELLET BIOPHANTOMS}

\section{A. Results}

Han et $a l^{14}$ performed high frequency (26-105 MHz) ultrasound BSC measurements on CHO cell pellet biophantoms. The actual radii of nuclear and whole CHO cells are $3.32 \pm 0.63$ $\mu \mathrm{m}$ and $6.71 \pm 0.86 \mu \mathrm{m}$, respectively. ${ }^{14}$ The solid curves in Fig. ??(a) represent some BSC raw data that were extracted from Figs. 9 and 10 of Ref. 14 using DataThief III software. The same procedure of parameter estimation presented in section III.D was applied to these data, except that we used different values for some known parameters: the true cell radius mean $\overline{r_{c}}=6.71 \mu \mathrm{m}$, the studied cell concentrations $\phi_{c}=(0.0017,0.0066,0.026,0.096,0.3$, 0.63 ) and the number of studied cell concentrations $N=6$.

The estimated parameters and the corresponding normalized errors computed with the three models FFSM, PM and SFM are summarized in Tables I and II (lines 4-6). Normalized errors were smaller with the polydisperse SFM and larger with the monodisperse PM. Figure ??(a) represents the BSCs results with the SFM in both monodisperse and polydisperse cases calculated by assuming the estimated scatterer size $\bar{r}^{*} \pm \mu^{*}$ and impedance contrast $\gamma_{z}^{*}$ given in Table II line 6. Figures ??(b) and ??(c) show the experimental and theoretical BSCs magnitude averaged in the frequency bandwidth (from 26 to $36 \mathrm{MHz}$ and from 26 to 105 $\mathrm{MHz}$ ) versus cell concentration. The $\mathrm{CHO}$ cell experiments present a complex $B S C_{\text {meas }}$ 
versus concentration relationship, which is nonlinear at low frequencies [Fig. ??(b)] and linear at high frequencies [Fig. ??(c)]. The polydisperse SFM was the model that better explained the $B S C_{\text {meas }}$ behaviors.

\section{B. Discussion}

The CHO cell experiments showed BSC smooth curves, which could not be modeled by a monodisperse model especially at high frequencies larger than $70 \mathrm{MHz}$ [see Fig. ?? and Fig. ??(a)]. That is why the errors obtained with the monodisperse modeling for the three models were about twice as large as those obtained with the polydisperse modeling [see Tables I and II lines 3-6].

It is interesting to observe that the error obtained with the FFSM was smaller than the error obtained with the PM in both monodiperse and polydisperse modeling cases (see Tables I and II lines 4 and 5). This is due to the linear relationship between the averaged $B S C_{\text {meas }}$ and the cell concentration at high frequency [see Fig. ??(c)], which can be easily reproduced with the FFSM. However, the observed $B S C_{\text {meas }}$ versus concentration relationship at low frequency cannot be modeled with the FFSM [see Fig. ??(b)]. The SFM was satisfactory to explain the BSC amplitude versus cell concentration whatever the frequency range studied. Indeed, the structure factor tends towards the packing factor in the low frequency range $(k r \rightarrow 0)$ and then oscillates around 1 for $k r \geq 1.5$ (see the solid lines in Fig. 14(a) in Ref. 21). That is why the SFM can display a nonlinear relationship for the BSCs averaged in the low 26-36 MHz frequency range and a linear relationship in the higher $26-105 \mathrm{MHz}$ frequency range [see the solid red lines in Fig. ??(b) and (c)].

For the polydisperse SFM giving the smallest errors, the estimated impedance contrast $\gamma_{z}^{*}=0.068$ agreed quite well with the expected value. The estimated radius of the fluid sphere was found equal to $5.47 \mu \mathrm{m}$, which is close to the true whole cell radius of $6.71 \mu \mathrm{m}$, i.e. relative error of $18 \%$. However, the correspondence between the true $\mathrm{CHO}$ cellular structures and the estimated radii was less accurate than the correspondence obtained with 
the K562 cells. The fluid-filled sphere might be less reliable for modeling a single cell in terms of shape and structure at high frequencies. Indeed, the product $k r_{c}$ was less than 1 $\left(0.26 \leq k r_{c} \leq 1.11\right.$ in the $10-42 \mathrm{MHz}$ frequency range) for the K562 cell experiments against $0.73 \leq k r_{c} \leq 2.95$ in the $26-105 \mathrm{MHz}$ frequency range for the $\mathrm{CHO}$ cell experiments. To confirm this hypothesis, the procedure of parameter estimation was applied in a reduced frequency bandwidth of 26-56 MHz. The upper frequency limit of $56 \mathrm{MHz}$ corresponds to the average of BSC peaks and to a product $k \overline{r_{c}}=1.5$. Tables I and II lines 7-9 give the estimated parameters and Fig. ?? represents the corresponding averaged BSCs versus the cell concentration for the estimation procedure performed in the 26-56 MHz frequency bandwidth. Normalized errors were smaller with the polydisperse SFM and larger with the FFSM, as previously obtained with the K562 cell study. For the polydisperse SFM giving the smallest errors, the radius of the fluid sphere was found equal to $6.04 \mu \mathrm{m}$ and match better the true $\mathrm{CHO}$ whole cell radius with a relative error of $10 \%$.

It is important to emphasize that cell pellet biophantoms mimic tumors only for high cellular concentration $\left(\phi_{c} \geq 0.3\right)$. Frequency dependent BSC from dense cell pellets exhibit similar frequency dependent BSC as xenograft tumors from the same cell line (see for example Fig. 5.3 in Ref. 35). So concentrated cell pellet biophantoms allow the investigation of the basics of the biophysical mechanisms of scattering, since a dense cell pellet constitutes a simplified version of a real tumor. The results obtained with the K562 cells (in the 10-42 $\mathrm{MHz}$ frequency bandwidth) and with the $\mathrm{CHO}$ cells (in the 26-56 MHz frequency bandwidth) suggest that the polydisperse SFM is an adequate model for QUS characterization of tumors with high cellular content. However, further study should be conducted on in vivo tumors to confirm the added value of the SFM. Indeed, tumors have more complex structures than cell pellets. The extracellular matrix, the blood microvessels and the tumor heterogeneity (with proliferating and necrotic cell type regions) may play a role in tumor backscatter, as shown by Han et al. ${ }^{36}$ At the moment, the SFM is an improvement over the FFSM for modeling high cellular content in simple tumor composed of a single cell line. 
Future work should focus on taking into account the heterogeneity of cell types as well as other structures such as blood microvessels.

\section{CONCLUSION}

Three scattering models were examined in view of explaining the experimental BSCs from cell pellet biophantoms at different cell concentrations with a single set of structural and acoustic parameters. Both K562 and CHO cell studies revealed that the FFSM and PM are insufficient to model the complex behavior of the $B S C_{\text {meas }}$ and that the polydisperse $\mathrm{SFM}$ is the model that better explains the behavior of $B S C_{\text {meas }}$. The impedance and size estimated with the polydisperse SFM are satisfactory: the relative impedance contrast estimates seem to be in a reasonable range of values and the fluid sphere radii match the true whole cell radii for both K562 and CHO cell studies. This finding shows that the whole cell plays a major role in the BSC behavior for the K562 and CHO cells studied. Note that, for these two cell lines, most of the cell volume is occupied by the cytoplasm $(71 \%$ for the K562 cell and $88 \%$ for the CHO cell). Future studies should focus on similar experiments with different kinds of cells with different nucleus to cell volume ratios.

\section{Acknowledgments}

This work was supported by the French Agence Nationale de la Recherche (ANR) under grant ANR Tecsan 11-008-01, Canceropole PACA and CNRS. The author would like to thank Eric Debieu and Stephan Devic from the Laboratory of Mechanics and Acoustics (LMA - CNRS) for their technical assistance.

\section{References}

1 F. L. Lizzi, M. Greenebaum, E. J. Feleppa, and M. Elbaum, "Theoretical framework for spectrum analysis in ultrasonic tissue characterization", J. Acoust. Soc. Am. 73, 1366$1373(1983)$. 
${ }^{2}$ F. L. Lizzi, M. Ostromogilsky, E. J. Feleppa, M. C. Rorke, and M. M. Yaremko, "Relationship of ultrasonic spectral parameters to features of tissue microstructure", IEEE Trans. Ultrason. Ferroelect. Freq. Contr. 33, 319-329 (1986).

3 M. C. Kolios, G. J. Czarnota, M. Lee, J. W. Hunt and M. D. Sherar, "Ultrasonic spectral parameter characterization of apoptosis", Ultrasound Med. Biol. 28, 589-597 (2002).

4 E. J. Feleppa, F. L. Lizzi, D. J. Coleman, and M. M. Yaremko, "Diagnostic spectrum analysis in ophthalmology: a physical perspective", Ultrasound Med. Biol. 12, 623-631 (1986).

5 E. J. Feleppa, T. Liu, A. Kalisz, M. C. Shao, N. Fleshner, and V. Reuter, "Ultrasonic spectral-parameter imaging of the prostate", Int. J. Imag. Syst. Technol. 8, 11-25 (1997).

${ }^{6}$ M. L. Oelze, J. F. Zachary and W. D. O’Brien, "Characterization of tissue microstructure using ultrasonic backscatter: Theory and technique for optimization using a Gaussian form factor", J. Acoust. Soc. Am. 112, 1202-1211 (2002)

7 M. L. Oelze, W. D. O’Brien, J. P. Blue, and J. F. Zachary, "Differentiation and characterization of rat mammary fibroadenomas and $4 \mathrm{~T} 1$ mouse carcinomas using quantitative ultrasound imaging", IEEE Trans. Med. Imaging 23, 764-771 (2004).

8 J. Mamou, A. Coron, M. Hata, J. Machi, E. Yanagihara, P. Laugier and E. Feleppa, "Three-dimensional high-frequency characterization of cancerous lymph nodes", Ultrasound Med. Biol. 36, 361-375 (2010).

9 V. C. Anderson, "Sound scattering from a fluid sphere", J. Acoust. Soc. Am. 22, 426-431 (1950).

10 M. L. Oelze and W. D. O'Brien, "Application of three scattering models to characterization of of solid tumors in mice", Ultrasonic Imaging 28, 83-96 (2006).

36 A. Han, R. Abuhabsah, J. P. Blue, S. Sarwate and W. D. O'Brien, "The measurement of ultrasound backscattering from cell pellet biophantoms and tumors ex vivo", J. Acoust. Soc. Am. 134, 686-693 (2013).

12 J. McNew, R. Lavarello and W. D. OBrien, "Sound scattering from two concentric fluid spheres (L)", J. Acoust. Soc. Am. 125, 1-4 (2009). 
13 M. Teisseire, A. Han, R. Abuhabsah, J. P. Blue, Jr., S. Sarwate and W. D. O'Brien, "Ultrasonic backscatter coefficient quantitative estimates from chinese hamster ovary cell pellet biophantoms", J. Acoust. Soc. Am. 128, 3175-3180 (2010).

14 A. Han, R. Abuhabsah, J. P. Blue, S. Sarwate and W. D. O’Brien, "Ultrasonic backscatter coefficient quantitative estimates from high-concentration Chinese hamster ovary cell pellet biophantoms", J. Acoust. Soc. Am. 130, 4139-4147 (2011).

15 J. S. Pedersen, "Determination of size distributions from small-angle scattering data for systems with effective hard-sphere interactions", J. Appl. Cryt. 27, 595-608 (1994).

16 J. S. Pedersen, "Analysis of small-angle scattering data from colloids and polymer solutions: modeling and lest-squares fitting", Advances in Colloid and Interface Science 70, 171-210 (1997).

17 R. M. Vlad, R. K. Saha, N. M. Alajez, S. Ranieari, G. J. Czarnota and M. C. Kolios, "An increase in cellular size variance contributes to the increase in ultrasound backscatter during cell death", Ultrasound in Medicine \& Biology 9, 1546-1558 (2010).

18 D. Savery and G. Cloutier, "A point process approach to assess the frequency dependence of ultrasound backscattering by aggregating red blood cells", J. Acoust. Soc. Am. 110, 3252-3262 (2001).

19 I. Fontaine, D. Savery and G. Cloutier, "Simulation of ultrasound backscattering by red blood cell aggregates: effect of shear rate and anisotropy", Biophysical Journal 82, 1696-1710 (2002).

20 V. Twersky, "Low-frequency scattering by correlated distributions of randomly oriented particles", J. Acoust. Soc. Am. 81 1609-1618 (1987).

21 E. Franceschini and R. Guillermin, "Experimental assessment of four ultrasound scattering models for characterizing concentrated tissue-mimicking phantoms", J. Acoust. Soc. Am. 132, 3735-3747 (2012).

${ }^{22}$ R. E. Baddour, M. D. Sherar, MD, J. W. Hunt, G. J. Czarnota, M. C. Kolios, "Highfrequency ultrasound scattering from microspheres and single cells", J. Acoust. Soc. Am. 117, 934-943 (2005). 
23 R. E. Baddour and M. C. Kolios, "The fluid and elastic nature of nucleated cells: implications from the cellular backscatter response", J. Acoust. Soc. Am. 121, EL16-22 (2007).

24 O. Falou, M. Rui, A. E. Kaffas, J. C. Kumaradas, and M. C. Kolios, "The measurement of ultrasound scattering from individual micron-sized objects and its application in single cell scattering," J. Acoust. Soc. Am. 128, 894-902 (2010).

25 J. J. Faran, "Sound Scattering by Solid Cylinders and Spheres", J. Acoust. Soc. Amer. 121, 405-418 (1951).

${ }^{26}$ L. R. Taggart, R. E. Baddour, A. Giles, G. J. Czarnota and M. C. Kolios, "Ultrasonic characterization of whole cells and isolated nuclei", Ultrasound Med. Biol. 33, 389-401 (2007).

27 M. S. Wertheim, "Exact solution of the Percus-Yevick integral equation for hard spheres", Physical Review Letters 10, 321-323 (1963).

28 N. E. Berger, R. J. Lucas and V. Twersky, "Polydisperse scattering theory and comparisons with data for red blood cells", J. Acoust. Soc. Am. 89, 1394-1401 (1991).

29 M. E. Greenberg and T. P. Bender, "Identification of newly transcribed RNA", in Current protocols in molecular biology (2007), Chap. 4:Unit 4.10. doi: 10.1002/0471142727.mb0410s78.

30 F. A. Duck, "Physical Properties of Tissue: A Comprehensive Reference Book", (Academic Press, London, 1990), Chap. 4, p. 95.

31 Yao L. X., Zagzebski J. A. and Madsen E. L., "Backscatter coefficient measurements using a reference phantom to extract depth-dependent instrumentation factors". Ultrasonic Imaging 12, 58-70 (1990).

32 S. H. Wang and K. K. Shung, "An approach for measuring ultrasonic backscattering from biological tissues with focused transducers, IEEE Trans. Biomed. Eng. 44, 549554 (1997).

33 Franceschini E., Metzger B. and Cloutier G., "Forward problem study of an effective medium model for ultrasound blood characterization", IEEE Trans. on Ultrason., Ferroelect., Freq. Contr. 58, 2668-2679 (2011). 
34 J. Bereither-Hahn, "Computer assisted microscope interferometry by image analysis of living cells", Proc. Clin. Biol. Res. 196, 27-44 (1985).

35 Falou O., Sadeghi-Naini A., Al-Mahrouki A., Kolios M. C., Czarnota G., "Quantittaive Ultrasound and Cell Death", in Quantitative Ultrasound in Soft Tissues, edited by J. Mamou and M. L. Oelze (Springer 2013), Chap. 5, pp. 95-115.

36 Han A., Abuhabsah R., Miller R. J., Sarwate S. and OBrien W. D., "The measurement of ultrasound backscattering from cell pellet biophantoms and tumors ex vivo", J. Acoust. Soc. Am. 134, 686-693 (2013). 


\section{TABLE CAPTIONS}

Table I. Summary of the estimated parameters given by the FFSM, PM and SFM in the monodisperse case.

Table II. Summary of the estimated parameters given by the FFSM, PM and SFM in the polydisperse case. 
TABLE I.

\begin{tabular}{|c|c|c|c|c|c|c|}
\hline & $\begin{array}{c}\text { Cell } \\
\text { line }\end{array}$ & $\begin{array}{c}\text { Frequency } \\
\text { bandwidth }\end{array}$ & Models & $r^{*}$ & $\left|\gamma_{z}^{*}\right|$ & Normalized \\
errors
\end{tabular}


TABLE II.

\begin{tabular}{|c|c|c|c|c|c|c|}
\hline & $\begin{array}{c}\text { Cell } \\
\text { line }\end{array}$ & $\begin{array}{c}\text { Frequency } \\
\text { bandwidth }\end{array}$ & Models & $\begin{array}{c}\bar{r}^{*} \pm \mu^{*} \\
(\mu \mathrm{m})\end{array}$ & $\left|\gamma_{z}^{*}\right|$ & $\begin{array}{c}\text { Normalized } \\
\text { errors }\end{array}$ \\
\hline 1 & K562 & $10-42 \mathrm{MHz}$ & FFSM & $4.48 \pm 0.00$ & 0.112 & 0.116 \\
\hline 2 & K562 & $10-42 \mathrm{MHz}$ & PM & $4.03 \pm 1.15$ & 0.137 & 0.053 \\
\hline 3 & K562 & $10-42 \mathrm{MHz}$ & SFM & $6.40 \pm 1.44$ & 0.051 & 0.029 \\
\hline \hline 4 & $\mathrm{CHO}$ & $26-105 \mathrm{MHz}$ & FFSM & $5.04 \pm 1.18$ & 0.073 & 0.108 \\
\hline 5 & $\mathrm{CHO}$ & $26-105 \mathrm{MHz}$ & PM & $4.02 \pm 1.67$ & 0.101 & 0.183 \\
\hline 6 & $\mathrm{CHO}$ & $26-105 \mathrm{MHz}$ & SFM & $5.47 \pm 1.07$ & 0.068 & 0.077 \\
\hline \hline 7 & $\mathrm{CHO}$ & $26-56 \mathrm{MHz}$ & FFSM & $3.83 \pm 1.39$ & 0.094 & 0.196 \\
\hline 8 & $\mathrm{CHO}$ & $26-56 \mathrm{MHz}$ & PM & $3.67 \pm 1.56$ & 0.124 & 0.069 \\
\hline 9 & $\mathrm{CHO}$ & $26-56 \mathrm{MHz}$ & SFM & $6.04 \pm 1.11$ & 0.057 & 0.054 \\
\hline
\end{tabular}


Figure 1. (Color online) Theoretical BSCs computed with the FFSM, PM and SFM considering a monodisperse distribution of sphere radius $6.5 \mu \mathrm{m}$ or a polydisperse distribution with a Gaussian size distribution of $6.5 \pm 1.5 \mu \mathrm{m}$ with an impedance contrast $\gamma_{z}=0.05$, for three sphere concentrations of $0.006,0.06$ and 0.3 .

Figure 2. (a) and (b) Optical microscopy of isolated K-562 nuclei and whole K-562 cells. Scale bars represent $50 \mu \mathrm{m}$. (c) and (d) Radius distribution of isolated K-562 nuclei and whole K-562 cells. The dashed lines represent the Gaussian distribution curves that approximate the nuclear and whole cell radius distribution, respectively.

Figure 3. (Color online) Representative HE stained sections of cell pellet biophantoms at several concentrations of $0.03,0.06,0.18$ and 0.30 .

Figure 4. (Color online) (a) US probe and cell pellet biophantom in a well immersed in PBS. (b) B-mode images of the cell pellet biophantoms obtained with the $20 \mathrm{MHz}$ center frequency probe for two cell concentrations of 0.18 and 0.30 .

Figure 5. (Color online) (a) Measured BSCs averaged over six measurements performed with the $20-\mathrm{MHz}$ and $30-\mathrm{MHz}$ center frequency probes for cell concentrations of $0.006,0.03,0.06$ and 0.12 . There is one experimental study. (b) and (c) Measured BSCs averaged over six measurements performed with the $20-\mathrm{MHz}$ and $30-\mathrm{MHz}$ center frequency probes for different cell concentrations. For each concentration, the two curves correspond to two different experimental studies (i.e. the preparation of the cell pellet biophantoms as well as the corresponding US acquisition). Standard deviations are not shown to enhance reading.

Figure 6. (Color online) Measured BSCs (in solid lines) and BSCs computed with the polydisperse FFSM and the polydisperse PM (in dashed lines) by assuming the estimated scatterer size $\bar{r}^{*} \pm \mu^{*}$ and impedance contrast $\gamma_{z}^{*}$ (see values in Table II lines $1-2)$. 
Figure 7. (Color online) Measured BSCs (in solid lines) and BSCs computed with the SFM in both monodisperse and polydisperse cases (in dashed lines) by assuming the estimated scatterer size $\left(r^{*}\right.$ or $\bar{r}^{*} \pm \mu^{*}$, respectively) and impedance contrast $\gamma_{z}^{*}$ (see values in Table I line 3 and Table II line 3, respectively).

Figure 8. (Color online) Comparison between the measured and predicted mean BSCs versus the K562 cell concentration for the FFSM, PM and SFM. Note that the procedure of parameter estimation was performed in the $10-42 \mathrm{MHz}$ frequency bandwidth.

Figure 9. (Color online) Comparison between the measured and predicted mean BSCs versus the K562 cell concentration for the PM. Note that the procedure of parameter estimation was performed in the $10-15 \mathrm{MHz}$ frequency bandwidth.

Figure 10. (Color online) (a) Experimental BSCs of CHO cell pellet biophantoms from Ref. 14 (in solid lines) and BSCs computed with the monodisperse SFM (in dotted lines) and with the polydisperse SFM (in dashed lines) by assuming the estimated scatterer size $\bar{r}^{*} \pm \mu^{*}$ and impedance contrast $\gamma_{z}^{*}$ (see values in Table I line 9 and Table II line 9). (b) and (c) Comparison between the measured and predicted mean BSCs versus the cell concentration. Note that the procedure of parameter estimation was performed in the 26-105 MHz frequency bandwidth.

Figure 11. (Color online) Comparison between the measured and predicted mean BSCs versus the cell concentration for the FFSM, PM and SFM. Note that the procedure of parameter estimation was performed in the $26-56 \mathrm{MHz}$ frequency bandwidth. 